\title{
The absence of chiasma terminalization and inverted meiosis in males and females of Myrmus miriformis Fn. (Corizidae, Heteroptera)
}

\author{
SEPPO NOKKALA* \& CHRISTINA NOKKALA \\ Laboratory of Genetics, Department of Biology, University of Turku, FIN-20014 Turku, Finland
}

\begin{abstract}
Terminalization of chiasmata and orientation of bivalents were analysed in males and females of Myrmus miriformis Fn. (Corizidae, Heteroptera). In spermatogenesis, the frequencies of the largest autosome bivalent with medial, subterminal and terminal chiasmata were compared at diplotene, early and late diakinesis and metaphase I. The bivalent displayed a single subterminal or medial chiasma in nearly 90 per cent of cells at diplotene. The frequency of such bivalents was still over 80 per cent at metaphase I, indicating the absence of complete chiasma terminalization. It is possible that chiasmata do not terminalize at all. In oogenesis, all bivalents at metaphase I displayed subterminal or medial chiasmata, indicating that terminalization is also absent in females. In both males and females all bivalents co-orientated at metaphase $\mathrm{I}$, the telomeres of homologous chromosomes being orientated towards opposite spindle poles. Hence, they underwent prereduction in meiosis; no evidence for an inverted meiosis could be found.
\end{abstract}

Keywords: bivalent orientation, chiasma terminalization, Heteroptera, holokinetic chromosomes, inverted meiosis.

\section{Introduction}

The observation that chiasmata are reduced in number and more terminally located at metaphase I as compared to earlier meiotic stages led Darlington (1929) to propose his hypothesis of chiasma terminalization. According to Darlington (1929), chiasmata will move from their original locations towards telomeres during late meiotic prophase. However, recent observations obtained by more refined techniques have shown that in species carrying chromosomes with localized centromeres, the terminalization of chiasmata does not occur, or is at least a rare event (for review see Jones \& Tease, 1980; Jones, 1987).

In organisms with holokinetic chromosomes, complete chiasma terminalization has been generally considered as a regular feature of meiosis (John \& Lewis, 1966; White, 1973; John, 1976). However, this generalization is mainly based on observations on rather few species, made from slides prepared with older techniques allowing only limited analysis of

\footnotetext{
*Correspondence. E-mail: seppo.nokkala@utu.fi
}

chiasma location in bivalents at different meiotic stages. As a rule, in species with holokinetic chromosomes the bivalents are heavily condensed at late diakinesis and metaphase I making interpretation of their structure very difficult. Therefore, most conclusions on the orientational behaviour of bivalents are based on the orientation of their longitudinal axes in relation to the axis of the spindle. It has been generally assumed that if the longitudinal axis of a bivalent is parallel to the axis of the spindle at metaphase I, i.e. the bivalent orientates axially, then prereductional separation of half-bivalents will follow at anaphase I, and meiosis is of the conventional type. Alternatively, if the longitudinal axis of a bivalent is parallel to the equatorial plane at metaphase I, i.e. the bivalent orientates equatorially, then the half-bivalents will divide at anaphase I, and meiosis is of the postreductional or inverted type (White, 1973).

The inverted type of meiosis has been suggested to occur in a wide range of organisms with holokinetic chromosomes (for review see White, 1973). Among Heteroptera, autosomal bivalents usually undergo conventional meiosis in males (Ueshima, 
1979). According to Helenius (1952; see also Halkka, 1956) some autosomal bivalents are able to undergo either conventional or inverted meiosis depending on their orientation at metaphase I. In female Heteroptera, which have been much less studied, meiosis is reported to be of the inverted type in at least one family (Halkka, 1956).

Although the presence of terminal or completely terminalized chiasmata in bivalents is used as an indication of the type of meiosis in species with holokinetic chromosomes, no attempts have been made to analyse the occurrence of terminalization quantitatively in these organisms. In the present study, the existence of chiasma terminalization was studied in the heteropteran Myrmus miriformis Fn. (Corizidae). An improved technique of chromosome preparation was used, which allows the accurate determination of chiasma location in bivalents during condensation from diplotene to metaphase I in the male and metaphase $I$ in the female.

\section{Materials and methods}

Adult males and females of $M$. miriformis (Corizidae, Hemiptera) were captured in the wild in the vicinity of Turku. Preparations were made and stained by the method of Puro \& Nokkala (1977). Briefly, testes and ovaries were subjected to a hypotonic treatment for 8-11 min and fixed in 6:3:1-fixative. Slide preparation was facilitated by Feulgen hydrolysis and brief staining in Schiff's reagent. Air-dried slides were finally stained with 4 per cent Giemsa and mounted in Entellan.

\section{Results}

\section{Chiasma terminalization}

The chromosome number of $M$. miriformis is $2 n=13$ $(10 \mathrm{~A}+2 \mathrm{~m}+\mathrm{X0}$ ) (cf. Ueshima, 1979; Nokkala, 1985). The karyotype includes one large pair of autosomes which forms an easily recognizable bivalent in meiosis. By the method used, the location of chiasma(ta) in the bivalent can be determined accurately in all meiotic stages. The number of chiasmata and their location in the bivalent were scored at diplotene, early diakinesis, late diakinesis and metaphase I in cells from 12 males (Table 1). The bivalent was found to display only one chiasma in over 90 per cent of the cells. A bivalent with two chiasmata was found in about 5 per cent of the cells. Unexpectedly large autosomal univalents were also found at a constant, though low, frequency during all stages analysed.
The location of chiasma was determined as medial, subterminal or terminal in monochiasmate bivalents. Over 80 per cent of chiasmata were subterminal, and the frequencies of terminal and medial chiasmata were very low. The frequency of bivalents with subterminal or medial chiasmata was still over 80 per cent at metaphase I. Clearly, on the evidence of this bivalent, complete chiasma terminalization is absent in $M$. miriformis males.

The question remains if chiasmata terminalize at all. The frequency of bivalents with subterminal chiasmata was lower in cells at late diakinesis and metaphase I cells than in diplotene cells (Table 1). Also the frequency of bivalents with terminal chiasmata was higher in metaphase I cells than diplotene cells. However, the frequency of bivalents with medial chiasmata was not affected by condensation, indicating that factors other than genuine chiasma terminalization are responsible for the changes in number of subterminal and terminal chiasmata. Most likely, subterminal chiasmata are more easily scored in error as terminal when chromosomes become more condensed. Accordingly, we conclude that if a chiasma is formed subterminally in a bivalent, it may retain its original location through diplotene (Fig. 1a), early diakinesis (Fig. 1b), late diakinesis (Fig. 1c) and metaphase I (Fig. 1d,e).

In female meiosis, insufficient high-quality metaphase plate preparations were obtained for statistical analysis. However, in those plates analysed (Fig. 2), all bivalents showed subterminal or medial chiasmata. Thus chiasma terminalization in female meiosis, if it occurs at all, is incomplete.

\section{Bivalent orientation}

When it has a subterminal chiasma, the largest bivalent in $M$. miriformis males may orientate at metaphase I either so that its longitudinal axis is parallel to the axis of the spindle (Fig. 1d), or parallel to the equatorial plane (Fig. 1e). These two orientations are referred here as axial-like and equatorial-like, respectively, to distinguish them clearly from genuine axial and equatorial orientations as defined by White (1973). Axial-like orientation was nearly three times more common than equatorial-like orientation (Table 2). Both types of orientation can still be identified at anaphase I. Axial-like orientation results in a dyad with well separated chromatids held together by sister chromatid cohesion between the kinetically active telomeres (Fig. 1f). When orientation is equatorial-like (Fig. 1g), the basic structure of the dyad is essentially the same, with chromatids held together by sister chromatid cohe- 
Table 1 The number and location of chiasmata in the largest autosome bivalent in spermatocytes in Myrmus miriformis

\begin{tabular}{|c|c|c|c|c|c|c|}
\hline \multirow[b]{2}{*}{ Stage } & \multicolumn{3}{|c|}{ One chiasma } & \multirow[b]{2}{*}{ Two chiasmata } & \multirow[b]{2}{*}{ Univalents } & \multirow[b]{2}{*}{ No. of cells } \\
\hline & Terminal & Subterminal & Medial & & & \\
\hline Diplotene & $18(4.1)$ & $371(85.1)$ & $21(4.8)$ & $22(5.0)$ & $4(0.9)$ & 436 \\
\hline Early diak. (\%) & $\begin{array}{c}25(4.6) \\
\mathrm{NS}^{1}\end{array}$ & $\begin{array}{c}478(87.4) \\
\text { NS }\end{array}$ & $\begin{array}{c}16(2.9) \\
\text { NS }\end{array}$ & $\begin{array}{c}26(4.8) \\
\text { NS }\end{array}$ & $\begin{array}{c}2(0.4) \\
\text { NS }\end{array}$ & 547 \\
\hline Late diak. $(\%)$ & $\begin{array}{c}40(8.8) \\
t=2.75^{* *}\end{array}$ & $\begin{array}{c}363(79.6) \\
t=2.16^{*}\end{array}$ & $\begin{array}{c}17(3.7) \\
\text { NS }\end{array}$ & $\begin{array}{c}32(7.0) \\
\text { NS }\end{array}$ & $\begin{array}{c}4(0.9) \\
\text { NS }\end{array}$ & 456 \\
\hline Metaphase I & $\begin{array}{c}28(7.0) \\
t=1.82^{*}\end{array}$ & $\begin{array}{l}306(76.7) \\
t=3.09^{* *}\end{array}$ & $\begin{array}{c}28(7.0) \\
\text { NS }\end{array}$ & $\begin{array}{c}35(8.8) \\
t=2.16^{*}\end{array}$ & $\begin{array}{c}2(0.5) \\
\text { NS }\end{array}$ & 399 \\
\hline
\end{tabular}

${ }^{1}$ Difference from diplotene is indicated (binomial $t$-test, one-tailed).

Fig. 1 The behaviour of the largest autosome pair in male Myrmus miriformis. A bivalent with a subterminal chiasma at: (a) diplotene; (b) early diakinesis; (c) late diakinesis; (d,e) metaphase I; (f) late anaphase I; (g) early anaphase I.

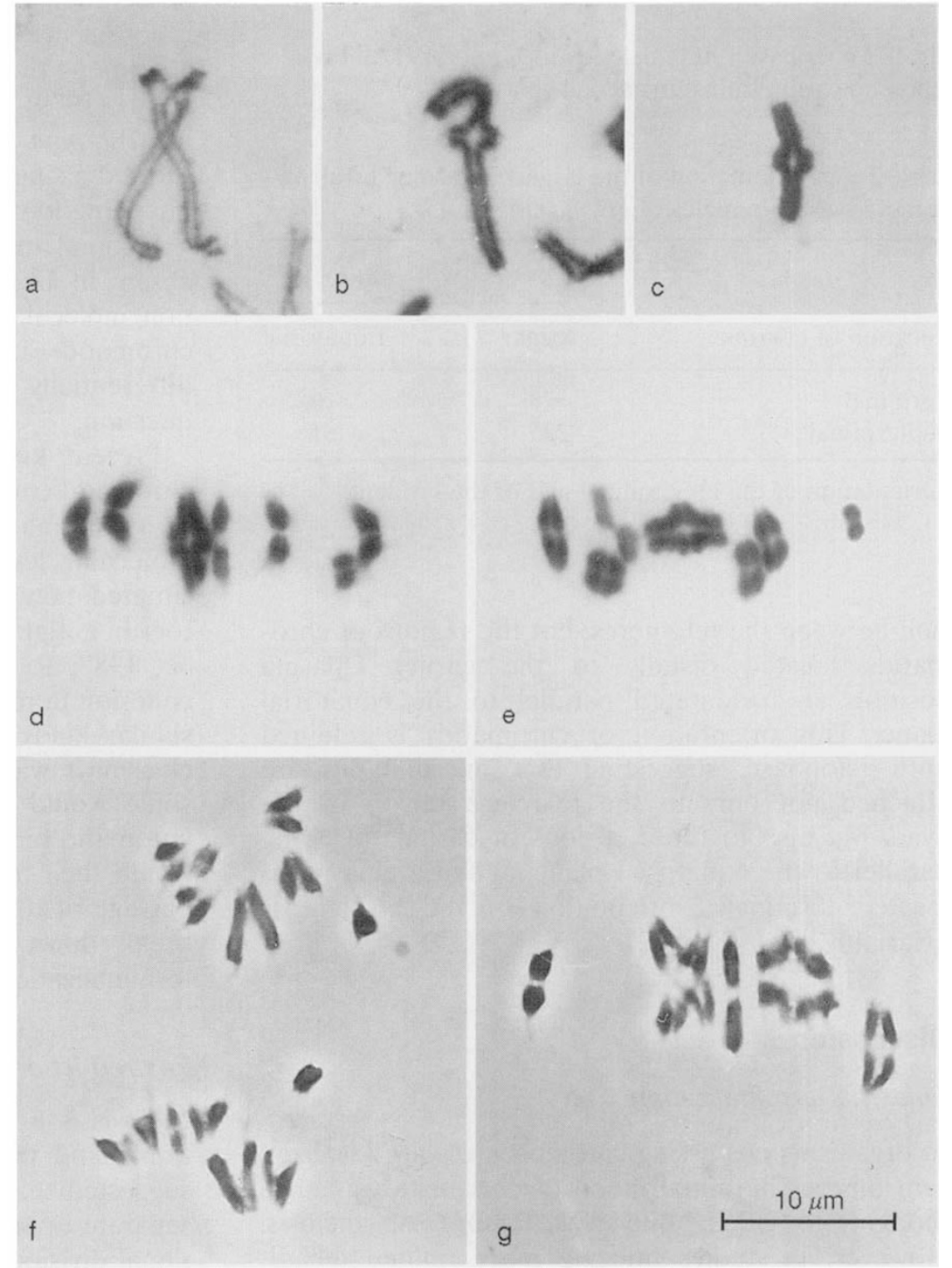

(c) The Genetical Society of Great Britain, Heredity, 78, 561-566. 


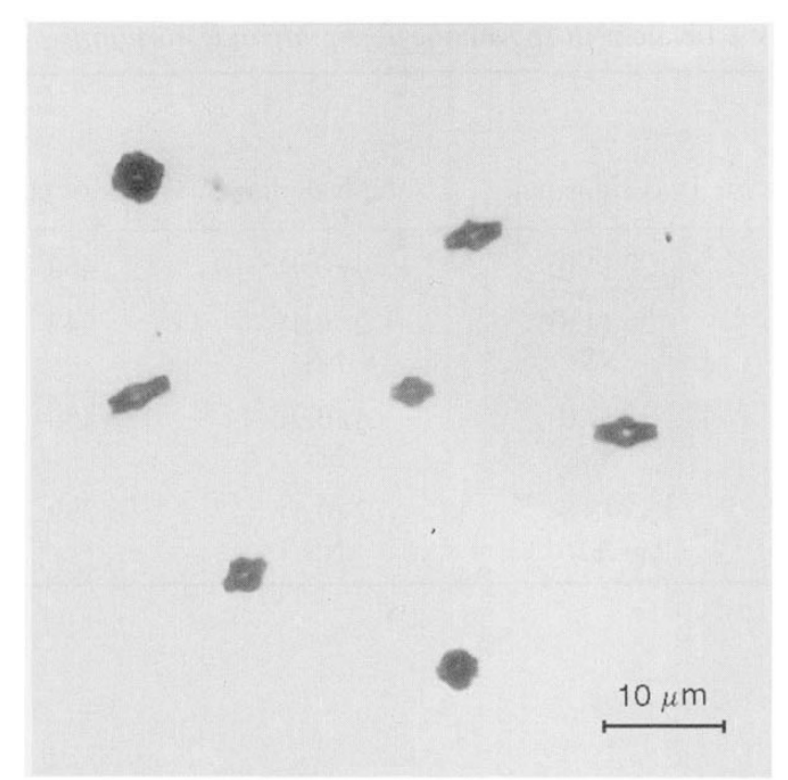

Fig. 2 Metaphase I in female Myrus miriformis. All bivalents show subterminal or medial chiasmata.

Table 2 The orientation of the largest autosome bivalent at metaphase I in males of Myrmus miriformis

\begin{tabular}{lcc}
\hline & \multicolumn{2}{c}{ Orientation $^{1}$} \\
\cline { 2 - 3 } Location of chiasma & Axial & Equatorial \\
\hline Terminal & 28 & 0 \\
Subterminal & 225 & 81 \\
\hline
\end{tabular}

${ }^{1}$ Orientation of the longitudinal axis of the bivalent.

sion between the telomeres, but the regions of chromatids located distally to the earlier chiasma position are orientated parallel to the equatorial plane. This orientation of chromatids is retained until telophase, suggesting that microtubules are attached not only to the telomere region of the dyad, but also to those regions of chromatids lying parallel to the equatorial plane. When a chiasma is located terminally, orientation of the bivalent is invariably axial (Table 2).

\section{Discussion}

\section{Chiasma terminalization}

In organisms possessing chromosomes with localized centromeres, terminalization of chiasmata was once thought to be a universal feature of meiosis. However, in studies utilizing more refined techni- ques to analyse chiasma terminalization, it has been shown that the phenomenon is absent from meiosis in a wide range of organisms (reviewed by Jones \& Tease, 1980; Jones, 1987). These observations have led to the opposite conclusion according to which there is no chiasma terminalization at all (cf. Jones, 1987). Accordingly, end-to-end association of chromosomes within a bivalent is explained as a consequence of extremely subterminal location of the chiasma or pseudoterminalization of a subterminal chiasma. In the case of terminal XY associations, at least some of these proved to be achiasmate in nature (Solari \& Ashley, 1977; Sharp, 1982; Wolf et al., 1988).

In organisms with holokinetic chromosomes, the occurrence of complete chiasma terminalization has been taken for granted (see White, 1973). The observations that complete chiasma terminalization is absent in both males and females of $M$. miriformis are among the first to challenge this rule. Previously, Solari (1979) observed that the holokinetic bivalents of Triatoma infestans (Reduviidae, Heteroptera) showed a single interstitial chiasma at all stages from diplotene to metaphase I.

Incomplete chiasma terminalization seems to be absent in $M$. miriformis males also, but high-resolution molecular labelling studies in which the sister chromatids in meiotic chromosomes are stained differentially (Tease, 1978) are needed to settle this question.

Present knowledge of the mechanism of sister chromatid cohesion suggests that the terminalization of a chiasma is a rather improbable process at the molecular level. In mitotic cells, specific proteins situated between sister chromatids are responsible for their tight apposition until anaphase (Cooke et al., 1987; Rattner et al., 1988). If sister chromatid cohesion in meiotic cells is based on the existence of similar kinds of proteins, then the movement of a chiasma towards the telomeric region of a chromosome would require the detachment of the sister chromatid binding proteins, and these same proteins would then have to bind nonsister chromatids after passage of the chiasma. Otherwise a bivalent would break down into two univalents during chiasma terminalization.

\section{Postreductional or inverted meiosis}

Meiosis is a process with two divisions, one reductional and the other equational. Originally, it was suggested that paternal and maternal chromosomes separate or segregate from each other in the reductional division, and divide in the equational division. 
In achiasmate meioses, reductional and equational divisions can still be distinguished in this way. In postreductional or inverted meiosis, the two divisions occur in an inverted sequence: equational followed by reductional. For example, in most Heteroptera studied, both $\mathrm{X}$ and $\mathrm{Y}$ chromosomes divide at the first meiotic division and segregate from each other at the second division (Ueshima, 1979). Even if the two chromosomes are physically linked via their telomeres by an achiasmatic linkage as in one aphid species (Blackman, 1985), the definitions of reductional and equational divisions are unambiguous.

In chiasmate meiosis, chromosomes will carry both maternal and paternal parts because of crossing-over, so the terms reductional and equational lose their significance (Oksala, 1943; Battaglia \& Boyes, 1955; White, 1973; Sybenga, 1981). As pointed out already by Oksala (1943), a morphological or cytological problem still exists, to make a distinction between divisions where whole chromosomes segregate, and where they divide, and he defined reductional and equational divisions accordingly. These definitions separate the two types of meioses which differ by a mechanism responsible for the integrity of a dyad. In the prereductional meiosis, the integrity of a dyad is based on sister chromatid cohesion, whereas in the postreductional meiosis the two chromatids are to be held together end-to-end by 'half a terminal chiasma'.

When Oksala's definitions are applied to holokinetic chromosomes, morphological postreduction can be said to occur only if: (i) a chiasma in a bivalent is terminal or completely terminalized; (ii) the bivalent shows equatorial orientation on the spindle of the first meiotic division. In $M$. miriformis, bivalents displaying a terminal chiasma orientate axially at the first meiotic division. Thus whole chromosomes will segregate at anaphase I, i.e. these bivalents undergo morphological prereduction. Furthermore, bivalents displaying a nonterminal chiasma, quite independent of chiasma location, result in genuine dyads or whole chromosomes at anaphase I. Consequently, they will also undergo prereduction both in male and female meiosis. Hence, there was no evidence for an inverted sequence in $M$. miriformis, either at the level of an individual bivalent, or in meiosis as a whole.

Earlier observations suggesting that in males of Heteroptera a bivalent may orientate either so that its long axis is parallel to the axis of the spindle, or parallel to the equatorial plane (Ekblom, 1934; Helenius, 1952), are apparently based on the behaviour of bivalents displaying a subterminal chiasma.
As a result of the dikinetic nature of holokinetic chromosomes in chiasmate meioses (Camacho et al., 1985; Nokkala, 1985; Albertson \& Thomson, 1993), these bivalents are able to orientate spatially in two different ways at metaphase I, but nevertheless show only one orientational type on the spindle. How should this orientation be described? The terms axial and equatorial are excluded, because these terms have been defined by White (1973) for bivalents with a terminal or terminalized chiasma. In our opinion the term co-orientation can be used, because the telomeres of homologous chromosomes are orientated towards opposite poles. It should be noted, however, that chromosome regions distal to the earlier chiasma position display auto-orientation. This auto-orientation is responsible for the parallel separation of these regions during anaphase I. To be logical, the terms axial and equatorial orientation should be replaced with co-orientation and autoorientation, respectively, the terms that have been used earlier in the context of holokinetic chromosomes (see Helenius, 1952; Hughes-Schrader \& Schrader, 1961; John \& Lewis, 1966).

Meiosis in the plant Luzula purpurea (Juncaceae) has been often cited as a typical example of postreduction. Luzula purpurea has only six chromosomes in the diploid set, and three bivalents are formed at meiosis. Interstitial or subterminal chiasmata are clearly visible in the bivalents still at diakinesis and metaphase I. The bivalents orientate with their longitudinal axes parallel to the equatorial plane at metaphase I. Half-bivalents separate in the parallel fashion at anaphase I, and earlier chiasma positions are still visible (Nordenskiöld, 1962). On the basis of these observations it is evident that the bivalents in $L$. purpurea co-orientate at metaphase I and are undergoing prereductional meiosis. The concept of the postreduction in L. purpurea is based on an erroneous interpretation of the arrangement of the chromatids in the half-bivalents. According to Nordenskiöld (1962), chromatids pointing to a pole in bivalents with subterminal chiasmata at metaphase I and anaphase I originate from homologous chromosomes, i.e. they would be nonsister chromatids, not sister chromatids as generally believed at present.

The present study suggests that all cases where inverted meiosis has been claimed to occur need to be reinvestigated. If the absence of chiasma terminalization is a general phenomenon in organisms with holokinetic chromosomes, the very existence of inverted meiosis must be questioned, because in many of these organisms, in Odonata (Oksala, 1943, 1945), and in Coccoidea (Hughes-Schrader, 1948) 
bivalents with nonterminal chiasmata are described in diakinetic nuclei.

\section{Acknowledgements}

This study was supported by the Academy of Finland.

\section{References}

ALBERTSON, D. G. AND THOMSON, J. N. 1993. Segregation of holokinetic chromosomes at meiosis in the nematode, Caenorhabditis elegans. Chromosome Res., 1, 15-26.

BATtAgliA, E. AND BOYES, J. W. 1955. Post-reductional meiosis: Its mechanism and causes. Caryologia, 8, 87-134.

BLACKMAN, R. L. 1985. Spermatogenesis in the aphid Amphorophora tuberculata (Homoptera, Aphididae). Chromosoma, 92, 357-362.

CAMACHO, J. P. M., BELDA, J. AND CABRERO, J. 1985. Meiotic behaviour of the holocentric chromosomes of Nezara viridula (Insecta, Heteroptera) analysed by C-banding and silver impregnation. Can. J. Genet. Cytol., 27, 491-497.

COOKE, C. A., HECK, M. M. S. AND EARNSHAW, W. c. 1987. The inner centromere protein (INCENP) antigens: movement from inner centromere to midbody during mitosis. J. Cell. Biol., 105, 2053-2067.

DARlington, C. D. 1929. Chromosome behaviour and structural hybridity in Tradescantiae. J. Genet., 21, 207-286.

EKBloM, т. 1934. Chromosomenstudien bei der Spermatogenese des Myrmus miriformis. Z. Zellf. Mikr. Anat., 21, 510-528.

HALKKA, o. 1956. Studies on mitotic and meiotic cell division in certain Hemiptera under normal and experimental conditions. Ann. Acad. Sci. Fenn. A. IV., 32, $1-80$.

HELENIUS, o. 1952. The mode of bivalent orientation in the Hemiptera. Hereditas, 38, 420-424.

Hughes-sChrader, s. 1948. Cytology of coccids (Coccoidea, Homoptera). Adv. Genet., 2, 127-203.

HUGHES-SCHRADER, S. AND SCHRADER, F. 1961. The kinetochore of the Hemiptera. Chromosoma, 12, 327-350.

JOHN, B. 1976. Myths and mechanisms of meiosis. Chromosoma, 54, 295-325.

JOHN, B. AND LEWIS, K. R. 1966. The meiotic system. Protoplasmatologia VI/F/1,1-335. Springer-Verlag, Vienna.

Jones, G. H. 1987. Chiasmata. In: Moens, P. B. (ed.)
Meiosis (Cell Biology: A Series of Monographs), pp. 213-244. Academic Press, Orlando, FL.

JONES, G. H. AND TEASE, C. 1980. Meiotic exchange analysis by molecular labelling. Chromosomes Today, 7, 114-125.

NOKKALA, s. 1985. Restriction of kinetic activity of holokinetic chromosomes in meiotic cells and its structural basis. Hereditas, 102, 85-88.

NORDENSKIÖLD, H. 1962. Studies of meiosis in Luzula purpurea. Hereditas, 48, 503-519.

OKSALA, T. 1943. Zytologische Studien an Odonaten. I. Chromosomenverhältnisse bei der Gattung Aeschna mit besonderer Berücksichtigung der postreduktionellen Teilung der Bivalente. Ann. Acad. Sci. Fenn. A. IV., 4, $1-63$.

OKSAlA, T. 1945. Zytologische Studien an Odonaten. III. Die Ovogenese. Ann. Acad. Sci. Fenn. A. IV., 9, 1-29.

PURO, J. AND NOKKALA, s. 1977. Meiotic segregation of chromosomes in Drosophila melanogaster oocytes. A cytological approach. Chromosoma, 63, 273-286.

RATTNER, J. B., KINGWELL, B. G. AND FRIZLER, M. J. 1988. Detection of distinct structural domains within the primary constriction using autoantibodies. Chromosoma, 96, 360-367.

SHARP, P. 1982. Sex chromosome pairing during meiosis in marsupials. Chromosoma, 86, 27-47.

SOLARI, A. J. 1979. Autosomal synaptonemal complexes and sex chromosomes without axes in Triatoma infestans (Reduviidae; Hemiptera). Chromosoma, 72, 225-240.

SOLARI, A. J. AND ASHLEY, T. 1977. Ultrastructure and behaviour of the achiasmatic telosynaptic XY pair of the sand rat (Psammomys obesus). Chromosoma, 62, 319-339.

SYBENGA, J. 1981. Specialization in the behaviour of chromosomes in the meiotic spindle. Genetica, 57, 143-151.

TEASE, C. 1978. Cytological detection of crossing-over in BUdR substituted meiotic chromosomes using the fluorescent plus Giemsa technique. Nature, 272, $823-824$.

ueshima, N. 1979. Animal Cytogenetics 3. Insecta 6. Hemiptera II: Heteroptera. Gebrüder Borntraeger, Berlin, Stuttgart.

white, M. J. D. 1973. Animal Cytology and Evolution, 3rd edn. Cambridge University Press, Cambridge.

Wolf, K. W., BAUMGarT, K. AND WINkING, H. 1988. Meiotic association and segregation of the achiasmatic giant sex chromosomes in the male field vole (Microtus agrestis). Chromosoma, 97, 124-133. 